\title{
ON INTEGRABLE AND BOUNDED AUTOMORPHIC FORMS. II
}

\author{
T. A. METZGER AND K. V. RAJESWARA RAO
}

\begin{abstract}
For a finitely generated Fuchsian group, every integrable automorphic form of arbitrary real dimension $<-2$ is bounded. If the group is, in addition, of second kind, then every integrable automorphic form of dimension -2 , with arbitrary factors of automorphy, is zero.
\end{abstract}

1. Introduction. Throughout, $\Gamma$ denotes a Fuchsian group acting on the unit disc $U$ of the complex plane. For any given real number $q$, we choose and fix, once and for all, a system $\rho(q, T, z)(z \in U, T \in \Gamma)$ of factors of automorphy of dimension $-2 q$ belonging to $\Gamma$ (cf. [3]). Note that, if $q$ is an integer, $\rho(q, T, z)=\chi(T) T^{\prime}(z)^{q}$, where $\chi$ is a character of $\Gamma$.

Let $\Omega$ be a Poincaré normal polygon of $\Gamma$ centered at a point in $U$ not fixed by $\Gamma$. Denote by $A_{q}(\Gamma)$ the space of integrable, holomorphic automorphic forms of dimension $-2 q$, and, by $B_{q}(\Gamma)$, the space of bounded, holomorphic automorphic forms of dimension $-2 q$ (cf. [3]). We prove:

THEOREM 1. Let $\Gamma$ be finitely generated. Then $A_{q}(\Gamma) \subset B_{q}(\Gamma)$ for $q>1$.

THEOREM 2. Let $\Gamma$ be finitely generated and be of second kind. Then $A_{1}(\Gamma)=\{0\}$.

Remarks. In the case where $q$ is an integer and $\rho(q, T, z)$ are the standard factors of automorphy $T^{\prime}(z)^{q}$, Theorem 1 was proved by Drasin and Earle [1] by an entirely different method. For arbitrary real $q$ and arbitrary $\rho$, Theorem 1 was established in [3] under the additional hypothesis that $\Gamma$ contains no parabolic elements. Theorem 2 is proved here by reducing it to the already established (cf. [3]) special case of standard factors of automorphy $\rho(1, T, z)=T^{\prime}(z)$. Thus, the present note is in the nature of an addendum to [3].

Received by the editors May 20, 1971.

AMS 1969 subject classifications. Primary 3049; Secondary 3045, 3085.

Key words and phrases. Fuchsian group, finitely generated, factors of automorphy, character, automorphic forms, bounded, integrable.

1 The authors were supported, in part, by the National Science Foundation.

(c) American Mathematical Society 1972 
2. Proof of Theorem 1. For $q>1$ and $z, \zeta$ in $U$, let

$$
K(z, \zeta)=\pi^{-1}(2 q-1)(1-z \bar{\zeta})^{-2 q}
$$

where $K$ is analytic in $z$ and $K(0, \zeta)>0$. By Theorem 1 of [3], it suffices to show that

$$
\sup _{z \in U}\left(1-|z|^{2}\right) \alpha(z, z)<\infty
$$

where

$$
\alpha(z, \zeta) \equiv \sum_{T \in \Gamma} \rho(q, T, z) K(T z, \zeta)
$$

Let $H^{\infty}$ be the Banach space of bounded analytic functions on $U$ with the supremum norm and $E$ be the Banach space of all holomorphic automorphic forms $F$ of dimension $-2 q$ such that the norm

$$
\|F\|=\sup _{z \in \Omega}|F(z)|<\infty .
$$

For $f$ in $H^{\infty}$, consider the Poincaré series

$$
\theta f(z)=\sum_{T \in \Gamma} \rho(q, T, z) f(T z) .
$$

Knopp [2, Proposition 3] proved that, if $f \in H^{\infty}$, then $\theta f \in E$. Standard arguments show that convergence of a sequence in norm in either of the spaces $H^{\infty}$ and $E$ implies uniform convergence on compact subsets of $U$. This readily implies that the map $f \rightarrow \theta f$ is a closed linear map of $H^{\infty}$ into $E$. The closed graph theorem then implies that $\theta: H^{\infty} \rightarrow E$ is a bounded linear map, i.e., there exists a constant $M<\infty$ such that, for all $f$ in $H^{\infty}$,

$$
|\theta f(z)| \leqq M \sup _{t \in U}|f(t)|, \quad z \in \Omega .
$$

Applying this to $f(t)=K(t, \zeta)$, we conclude that

$$
|\alpha(z, \zeta)| \leqq M \pi^{-1}(2 q-1) \frac{2^{2 q}}{\left(1-|\zeta|^{2}\right)^{2 q}}, \quad z \in \Omega, \zeta \in \Omega .
$$

Setting $\zeta=z$, we obtain the existence of a constant $N<\infty$ such that

$$
\beta(z) \equiv \alpha(z, z)\left(1-|z|^{2}\right)^{2 q} \leqq N, \quad z \in \Omega .
$$

Since, for all $T$ in $\Gamma, \beta \circ T=\beta$ and $\Omega$ is a fundamental region for $\Gamma$, (2.1) follows and the theorem is proved.

3. An auxiliary result. The proof of Theorem 2 is based on the following result of independent interest. 
THEOREM 3. Let $\Gamma$ be finitely generated and be of second kind. For any character $\lambda$ of $\Gamma$, there exists a function $g$ bounded and analytic on $U$, not identically zero on $U$ and satisfying

$$
\lambda(T) \cdot(g \circ T)=g, \quad T \in \Gamma .
$$

Proof. If the theorem is true for $\Gamma$, then it is true for every conjugate of $\Gamma$ (in the full group of conformal selfmaps of $U$ ). Hence we can and do assume that the origin in $U$ is not fixed by $\Gamma$ and let $\Omega$ be the normal polygon centered at the origin.

Let $f$ be a holomorphic automorphic form of dimension -2 belonging to $\Gamma$ with factors of automorphy $\lambda(T) \cdot T^{\prime}(z)$ :

$$
\lambda(T) \cdot T^{\prime} \cdot(f \circ T)=f, \quad T \in \Gamma .
$$

Let $g$ be the antiderivative of $f$ satisfying $g(0)=0$. (3.2) implies, for each $T$ in $\Gamma$, the existence of a constant $C(T, f)$ such that

$$
\lambda(T) \cdot(g \circ T)=g+C(T, f), \quad T \in \Gamma .
$$

It is readily verified that, for all $T_{1}, T_{2}$ in $\Gamma$,

$$
C\left(T_{1} T_{2}, f\right)=\lambda\left(T_{2}\right) \cdot C\left(T_{1}, f\right)+C\left(T_{2}, f\right) .
$$

It follows that the set $\{T \in \Gamma \mid C(T, f)=0\}$ is a subgroup of $\Gamma$. Since $\Gamma$ is finitely generated, the theorem will be proved if there exists a nontrivial $f$ such that the corresponding $g$ is bounded on $U$ and is such that

$$
C\left(S_{j}, f\right)=0, \quad j=1,2, \cdots, m,
$$

where $\left\{S_{1}, \cdots, S_{m}\right\}$ is a generating set for $\Gamma$. We choose $f(z)=\theta p(z) \equiv$ $\sum_{T \in \Gamma} \lambda(T) T^{\prime}(z) p(T z)$ for a suitable polynomial $p=\sum_{i=0}^{n} a_{i} p_{i}$, where $p_{i}(z)=z^{i}$ and $n \geqq m$. Let $f_{i}=\theta p_{i}$. Since $\theta$ is a linear map and $h \rightarrow C(T, h)$ is linear, the condition (3.3) is equivalent to: $\sum_{i=0}^{n} a_{i} C\left(S_{j}, f_{i}\right)=0, j=1$, $2, \cdots, m$. These are $m$ linear equations in $n+1>m$ unknowns $a_{i}$ and hence they have a nontrivial solution $\left(a_{0}, \cdots, a_{n}\right)$. The corresponding $f$ satisfies (3.3). Also, remembering that $\Gamma$ is of the second kind and examining the behaviour of $f$ at the point at infinity (cf. [4, Proposition 3]), one sees that $f$ is not identically zero on $U$. Hence the corresponding $g \not \equiv 0$ on $U$ and satisfies (3.1). Since $p$ is bounded on $U$, Proposition 3 of Knopp [2] shows that $f=\theta p$ is bounded on $\Omega: \sup _{z \in \Omega}|f(z)| \equiv M<\infty$. Hence, for $z$ in $\Omega$,

$$
|g(z)|=\left|\int_{0}^{z} f(t) d t\right| \leqq M|z| \leqq M ;
$$

here, we have integrated along the radial segment and this is justified 
since $\Omega$ is non-Euclidean convex and the radial segment is the nonEuclidean line segment. Thus $g$ is bounded on $\Omega$. Since $\Omega$ is a fundamental region for $\Gamma$ and $|\lambda|=1$, (3.1) now implies that $g$ is bounded on $U$ thus completing the proof.

4. Proof of Theorem 2. Let $F \in A_{1}(\Gamma)$ and the factors of automorphy be $\rho(1, z, T)=\chi(T) T^{\prime}(z)$, where $\chi$ is a character of $\Gamma$. Thus $\chi(T) \cdot T^{\prime}$. $(F \circ T)=F$ and $\iint_{\Omega}|F(z)| d x d y /\left(1-|z|^{2}\right)<\infty$. Choose $g$ as in Theorem 3 above with $\lambda=\bar{\chi}$ and let $G=g \cdot F$. Then $T^{\prime} \cdot(G \circ T)=G$ for all $T$ in $\Gamma$ and thus $G$ is a holomorphic automorphic form of dimension -2 with the standard factors of automorphy $T^{\prime}(z)$. Moreover $G$ is an integrable form:

$$
\iint_{\Omega}|G(z)| \cdot \frac{d x d y}{1-|z|^{2}} \leqq \sup _{z \in U}|g(z)| \iint_{\Omega} \frac{|F(z)|}{1-|z|^{2}} d x d y<\infty .
$$

Hence, by Theorem 3 of [3], $G$ is identically zero on $U$. Since $g$ is not identically zero, it follows that $F$ is identically zero on $U$, thus establishing the theorem.

\section{REFERENCES}

1. D. Drasin and C. J. Earle, On the boundedness of automorphic forms, Proc. Amer. Math. Soc. 19 (1968), 1039-1042. MR 39 \#442.

2. M. I. Knopp, A corona theorem for automorphic forms and related results, Amer. J. Math. 91 (1969), 599-618. MR 40 \#4450.

3. T. A. Metzger and K. V. Rajeswara Rao, On integrable and bounded automorphic forms, Proc. Amer. Math. Soc. 28 (1971), 562-566.

4. K. V. Rajeswara Rao, Fuchsian groups of convergence type and Poincaré series of dimension -2, J. Math. Mech. 18 (1968/69), 629-644. MR 38 \#6065.

Department of Mathematics, Purdue University, Lafayette, Indiana 47907

Current address (Metzger): Department of Mathematics, Texas A \& M University, College Station, Texas 77843 